\title{
Management of Peptic Ulcer Bleeding Refractory to Endoscopic Treatment
}

\author{
Tung Yen Lee*, Cheng Cheng Tung \\ Division of General Surgery, Department of Surgery, Changhua Christian Hospital, Taiwan \\ Email: "69051@cch.org.tw
}

Received 29 April 2016; accepted 4 June 2016; published 7 June 2016

Copyright (C) 2016 by authors and Scientific Research Publishing Inc.

This work is licensed under the Creative Commons Attribution International License (CC BY).

http://creativecommons.org/licenses/by/4.0/

\section{(c) (i) Open Access}

\begin{abstract}
Background: Before the advent of transcatheter arterial embolization (TAE), emergency surgery was the only choice for patients with peptic ulcer bleeding refractory to endoscopic therapy. This study compared the effectiveness of TAE and surgery in patients with peptic ulcer bleeding refractory to endoscopic hemostasis. Materials and Methods: This was a retrospective analysis of 116 patients with peptic ulcer bleeding refractory to endoscopic treatment at our institution. Eighty-three cases were treated with surgery, and 33 cases were managed with TAE. Clinical outcomes were evaluated. Results: There were no differences between groups with respect to the mortality rate $(p>0.05)$, length of hospital stay, or medical diseases related to mortality. The TAE group exhibited a significantly higher rebleeding rate $(p<0.05)$. Rebleeding predominantly occurred in patients with type Ia peptic ulcers (Forrest classification) irrespective of the treatment approach. The rebleeding rates in such patents were $30.2 \%$ and $56.3 \%$ in the surgery and TAE groups, respectively. Patients with rebleeding after further therapy showed high mortality rates (68.6\%). The rebleeding rate was not significantly different between the subgroups of patients with type Ia lesions, although there was a higher mortality rate in the TAE group $(27.9 \%$ vs. $75 \%, p$ $=0.001$ ). Conclusions: TAE may be the first-choice therapy for patients with peptic ulcer bleeding refractory to endoscopic treatment, whereas emergency surgery may be used as an alternative in patients with type Ia bleeding at institutions with no 24-hour radiology service or when no experienced radiologist is available.
\end{abstract}

\section{Keywords}

Peptic Ulcer Bleeding, Surgery, Transcatheter Arterial Embolization

\section{Introduction}

Despite the improvements in perioperative management and endoscopy techniques, peptic ulcer bleeding (PUB) *Corresponding author. 
continues to be a major complication and life-threatening emergency, with a mortality rate of approximately $10 \%$ [1] [2]. Endoscopic treatment is the first-choice treatment for hemostasis and localizing the origin of bleeding. This approach followed by Helicobacter pylori eradication and the usage of a proton pump inhibitor achieves primary hemostasis in approximately $90 \%$ of cases and lowers the risk of rebleeding [3] [4]. Although endoscopic treatment in combination with a proton pump inhibitor is effective in most patients with PUB, further therapy is required in approximately $20 \%$ of such individuals because of ongoing or recurrent bleeding.

Before the advent of transcatheter arterial embolization (TAE), emergency surgery is the only choice for patients with PUB refractory to endoscopic therapy, although the morbidity and mortality rates are reported to range from $30 \%$ to $50 \%$ and from $20 \%$ to $50 \%$, respectively [5]-[7]. Since the introduction of TAE, some studies [8] [9] supported by the recommendations of an international consensus [10] suggest that this technique can be an alternative to surgery if it is performed by experienced radiologists.

Therefore, this study compares surgery and TAE as salvage therapies in patients with PUB refractory to endoscopic therapy.

\section{Materials and Methods}

We retrospectively reviewed the medical records of patients with PUB admitted to Changhua Christian Hospital between January 2009 and December 2013. A total of 1,393 patients presented with peptic ulcer with active bleeding, and endoscopic treatment was performed in all cases.

At the beginning of the study period, most patients were referred to surgery because of ongoing bleeding after endoscopic hemostasis. Patients received TAE mainly when a radiologist was available and if they were poor candidates for surgery after discussion among the surgeon, physician, and family. "Poor candidates" is the decision of family after discussion. These reasons include elderly, illness, more medical diseases and less invasion with TAE in family's concept.

Among the 116 (9.7\%) patients refractory to endoscopic treatment, emergency salvage therapies were performed with surgery $(n=83)$ and TAE $(n=33)$ for ongoing bleeding. These 116 patients were enrolled in the study, and their characteristics are summarized in Table 1.

Throughout the study period, all TAE procedures were scheduled during working hours. The procedure involved selective catheterization of the celiac trunk, superior mesenteric artery, and gastroduodenal artery with a 5-F catheter from the femoral approach. Patients with contrast extravasation received coil embolization. Blind embolization was not performed in 4 cases in which no contrast extravasation was found; these cases were excluded from analysis. The operative method involved duodenotomy and oversewing of the ulcer. Rebleeding was defined and diagnosed by abdominal computed tomography as a bleeding episode within 3 days after salvage therapy during the same hospital admission manifesting as fresh hematemesis or melena and requiring the transfusion of at least 2 units of packed red blood cells [7]. The following outcome parameters were analyzed: mortality, morbidity, rebleeding rate, and length of hospitalization.

Table 1. Patient characteristics.

\begin{tabular}{cccc}
\hline & Surgery & TAE & $p$ \\
\hline Age (years) & $n=83$ & $n=33$ & 0.020 \\
Male & $68.25 \pm 14.067$ (range: $28-94)$ & $61.27 \pm 14.976$ (range: $28-82)$ & $0.099^{\mathrm{a}}$ \\
Female & $63(75.9)$ & $20(60.6)$ & \\
Duodenal ulcer & $20(24.1)$ & $13(39.4)$ & $0.885^{\mathrm{a}}$ \\
Gastric ulcer & $49(59.0)$ & $19(57.6)$ & $0.744^{\mathrm{a}}$ \\
Heart diseases & $34(41.0)$ & $14(42.4)$ & $1.00^{\mathrm{b}}$ \\
COPD & $30(36.2)$ & $13(39.4)$ & $0.962^{\mathrm{a}}$ \\
Metabolic diseases & $4(4.8)$ & $9(27.0)$ & $3(9.1)$ \\
Uremia & $23(27.7)$ & $7(21.2)$ & $0.271^{\mathrm{b}}$ \\
Malignancy & $15(18.1)$ & $0.285^{\mathrm{a}}$ \\
\hline
\end{tabular}

Data are presented as mean \pm SD or $n$ (\%). ${ }^{a}$ Pearson's $\chi^{2}$ test; ${ }^{\mathrm{b}}$ Fisher's exact test; others: independent $t$-test. COPD: chronic obstructive pulmonary disease; TAE: transcatheter arterial embolization. 
This retrospective study was approved by the Institutional Review Board of Changhua Christian Hospital, which waived the need for patient informed consent.

\section{Statistical Analysis}

The data were compiled into a database. The patients were classified into 2 groups according to the salvage method used: the TAE and surgery groups. Pearson's $\chi^{2}$ test and Fisher's exact test were used to examine the differences in the characteristics and outcomes between groups. Other variables were analyzed using independent $t$-tests. Continuous variables are presented as mean \pm standard deviation (SD). The level of significance was set at $p<0.05$. Statistical analyses were performed with SPSS version 17.0 (SPSS Inc., Chicago, IL, USA).

\section{Results}

A total of 492 (35.3\%) female and 901 (64.7\%) male patients with a mean age of 68.7 years underwent endoscopic treatment. Hemostasis was successfully achieved in $91.7 \%$ of cases. Endoscopic treatment failed in 116 (8.3\%) patients, necessitating further salvage therapy included surgery and TAE; 83 and 33 were treated with surgery and TAE, respectively. The patients who received surgery were significantly older than those who underwent TAE (68.3 \pm 14.1 vs. $61.27 \pm 15.0$ years, $p<0.05$ ). There were no other differences between the surgery and TAE groups (Table 1 ).

Regarding outcomes, 16 (48.5\%) and 19 (22.9\%) patients experienced rebleeding in the TAE and surgery groups, respectively. The rebleeding rate was significantly higher in the TAE group $(p=0.007)$ (Table 2$)$. There were no significant differences between groups with respect to mortality rates, length of hospital stay, or mortality related to medical diseases, although the mortality rate tended to be higher in the TAE group (51.5\% vs. 38.6\%, $p=0.202$ ) (Table 3).

Complications were more common in the surgery group than the TAE group (49/83, 59\% vs. 7/33, 21.2\%) (Table 4). Complications were managed with conservative methods, and further surgical interventions were unnecessary. There were no procedure-related complications (e.g., cholecystitis, ischemic bowel, etc.) in the TAE group.

Table 2. Comparison of outcomes between the TAE and surgery groups.

\begin{tabular}{cccc}
\hline & Surgery & TAE & $p$ \\
\hline & $n=83$ & $n=33$ & \\
\hline Re-bleeding & $19(22.9)$ & $16(48.5)$ & $0.007^{\mathrm{a}}$ \\
Acute renal failure & $18(21.7)$ & $4(12.1)$ & $0.300^{\mathrm{b}}$ \\
Sepsis & $52(62.7)$ & $16(48.5)$ & 0.162 \\
ICU (days) & $20.39 \pm 21.744$ & $19.10 \pm 17.627$ & 0.807 \\
Hospitalization (days) & $(1-158)$ & $23.39 \pm 18.932$ & 0.103 \\
Alive & $33.06 \pm 31.589$ & $(1-76)$ & $0.202^{\mathrm{a}}$ \\
Dead & $(2-219)$ & $16(48.5)$ & $17(51.5)$ \\
\hline
\end{tabular}

Data are presented as mean \pm SD (range) or $n(\%) .{ }^{a}$ Pearson's $\chi^{2}$ test; ${ }^{b}$ Fisher's exact test; others: independent $t$-test. ICU: intensive care unit; TAE: transcatheter arterial embolization.

Table 3. Medical diseases and mortality.

\begin{tabular}{|c|c|c|c|}
\hline & Surgery & TAE & $p$ \\
\hline & $n=36$ & $n=16$ & \\
\hline Heart diseases & $16(44.4)$ & $5(31.3)$ & $0.371^{\mathrm{a}}$ \\
\hline COPD & $4(11.1)$ & $1(6.3)$ & $1.00^{\mathrm{b}}$ \\
\hline Metabolic disease & $11(30.6)$ & $5(31.3)$ & $0.960^{\mathrm{a}}$ \\
\hline Uremia & $7(19.4)$ & $2(12.5)$ & $0.704^{\mathrm{b}}$ \\
\hline Malignancy & $2(6.3)$ & $4(23.5)$ & $0.164^{\mathrm{b}}$ \\
\hline
\end{tabular}

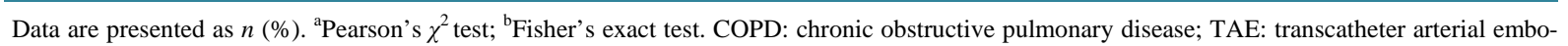
lization. 
Table 4. Complications.

\begin{tabular}{ccc}
\hline & Surgery & TAE \\
\hline & $n=49(49 / 83,59 \%)$ & $n=7(7 / 33,21.2 \%)$ \\
ARF & 24 & 5 \\
MOF & 2 & 1 \\
AMI & 0 & 0 \\
IAA & 10 & 0 \\
Leakage & 3 & 0 \\
Wound infection & 10 & 0 \\
\hline
\end{tabular}

ARF: acute renal failure; MOF: multiple organ failure; AMI: acute myocardial infarction; IAA: intra-abdominal abscess; TAE: transcatheter arterial embolization

\section{Rebleeding}

We further analyzed the patients with rebleeding in the surgery and TAE groups. Of the 43 and 16 patients with type Ia PUB (Forrest classification) in the surgery and TAE groups, rebleeding occurred in $13(30.2 \%)$ and 9 (56.3\%), respectively (Table 5). Twelve patients in each group died despite further salvage attempts including surgery, TAE, and endoscopy (Figure 1). Although the rebleeding rate was not significantly different between the surgery and TAE groups $(13 / 43,30.2 \%$ vs. $9 / 16,56.3 \%, p=0.066)$, the mortality rate was significantly higher in patients the TAE group who had type Ia lesions and rebleeding $(12 / 43,27.9 \%$ vs. $12 / 16,75 \%, p=$ $0.001)$. Rebleeding occurred predominantly in patients with type Ia lesions despite surgery $(12 / 19,63.2 \%)$ and TAE (9/16, 56.3\%). Accordingly, the mortality rate of rebleeding was $63.2 \%(12 / 19)$ and $75 \%(12 / 16)$ in the surgery and TAE groups, respectively. The overall mortality rate in patients with rebleeding was $68.6 \%$ (24/35).

\section{Discussion}

Although endoscopic treatment is the first-choice therapy for PUB, salvage treatment for ongoing or recurrent bleeding is required in approximately $10 \%-20 \%$ of patients, which is similar to the results in the present study. The management of PUB refractory to endoscopic hemostasis is very challenging. Therefore, selecting the best salvage therapy in such cases is imperative. Prompt intervention is essential for patient survival, because their clinical conditions deteriorate rapidly unless treatment is initiated immediately. Although surgery has traditionally been the only option, recently published articles propose TAE performed by an experienced radiologist with 24-hour availability as a feasible alternative [11] [12].

Although the technical and clinical success rates of TAE range from $52 \%$ to $99 \%$ and $50 \%$ to $76 \%$, respectively [13] [14], the rebleeding and mortality rates vary in the literature. For example, Eriksson et al. [12] compare the outcomes between TAE $(n=40)$ and surgery $(n=51)$ and report a relatively low mortality rate but higher rebleeding rate in the TAE group (3\% vs. $14 \%$ and $25 \%$ vs. $18 \%$, respectively); it should be noted that a skilled radiologist is available at their institution 24 hours per day. Meanwhile, Walsh et al. [15] report that only 26 of 50 (52\%) patients are successfully treated with TAE similar to ours.

Several factors may affect the success of angiographic embolization or lead to rebleeding, including the choice of embolic agents, type Ia PUB, endoscopic marking with a metallic clip, the use of a sandwich technique, and 24-hour availability of an experienced radiologist [14] [16]-[19]. In particular, marking with a metallic clip may reduce the incidence of rebleeding and improve outcomes in cases with no contrast extravasation, resulting in blind embolization. Furthermore, a sandwich technique has been suggested, in which both ends of the gastroduodenal artery are embolized to prevent retrograde bleeding from the superior mesenteric circulation.

The study focuses on the difference of salvage methods and institutions equipment. So, many factors such as coagulopathy, pre-procedure condition of patients, blood transfusion amount and length of time from diagnosis to salvage therapy are not analyzed herein. Because there is no 24-hour interventional radiology service at our institution, our patients undergo scheduled angiographic embolization during working hours. Furthermore, we do not routinely perform endoscopic marking with a metallic clip. A well-trained but relatively inexperienced radiologist performs embolization with coils and not combined agents, and the sandwich technique is not performed. These factors may have contributed to the relatively higher rebleeding incidence observed, thus weakening the present results. 
Table 5. Rebleeding in patients with type Ia lesions.

\begin{tabular}{|c|c|c|c|c|c|c|c|c|}
\hline \multicolumn{9}{|c|}{ Group } \\
\hline & & \multicolumn{2}{|c|}{ Surgery $(n=43)$} & \multicolumn{2}{|c|}{ TAE $(n=16)$} & \multicolumn{2}{|c|}{ Total $(n=59)$} & \multirow[t]{2}{*}{$p^{\mathrm{a}}$} \\
\hline & & $n$ & $\%$ & $n$ & $\%$ & $n$ & $\%$ & \\
\hline \multirow[t]{2}{*}{ Rebleeding } & No & 30 & 69.8 & 7 & 43.8 & 37 & 62.7 & 0.066 \\
\hline & Yes & 13 & 30.2 & 9 & 56.3 & 22 & 37.3 & \\
\hline \multirow[t]{2}{*}{ Mortality } & No & 31 & 72.1 & 4 & 25.0 & 35 & 59.3 & 0.001 \\
\hline & Yes & 12 & 27.9 & 12 & 75.0 & 24 & 40.7 & \\
\hline
\end{tabular}

${ }^{\mathrm{a}}$ Pearson's $\chi^{2}$ test.

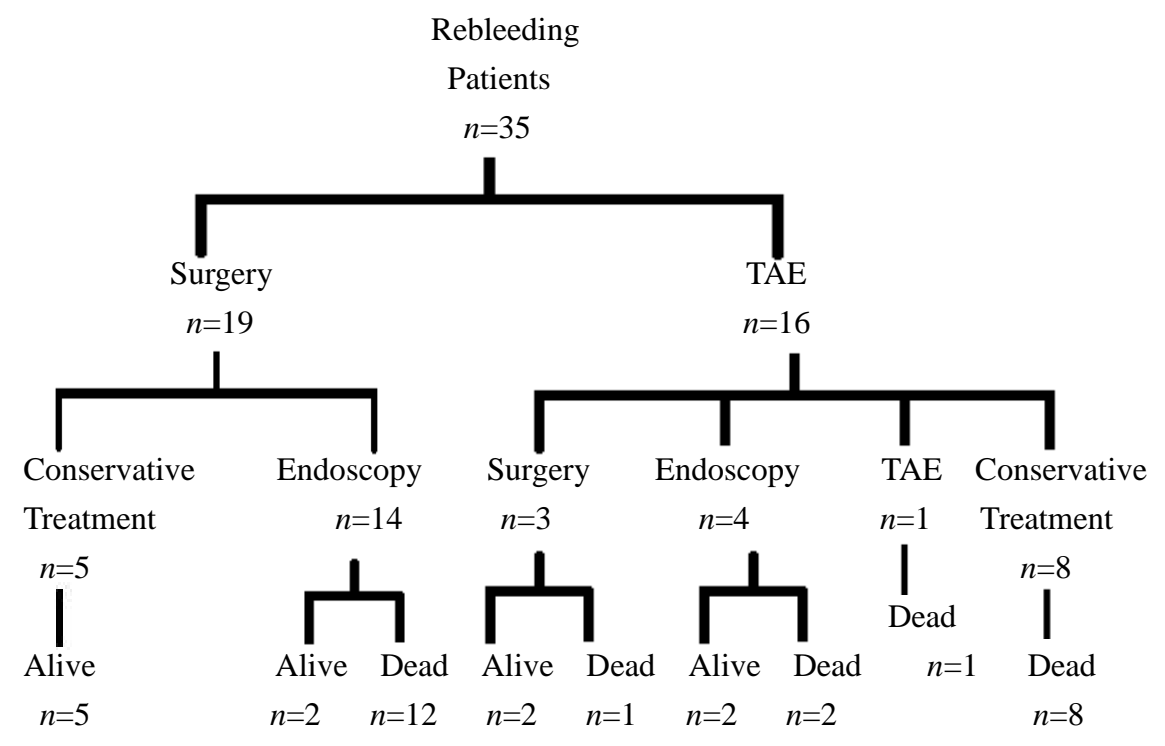

Figure 1. Patients with rebleeding in the surgery and transcatheter arterial embolization (TAE) groups.

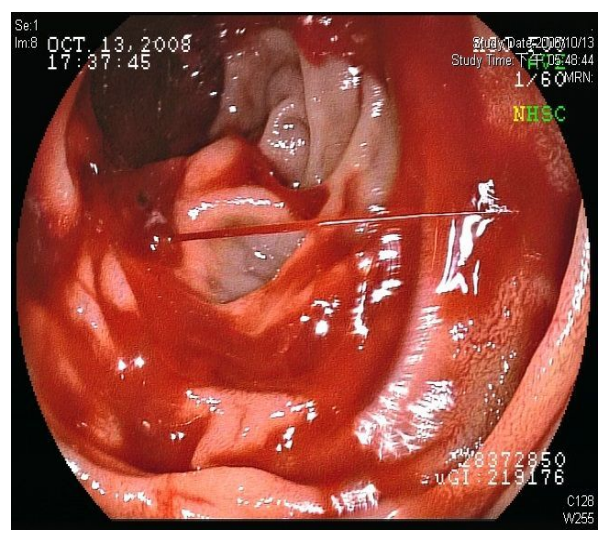

Figure 2. Type Ia ulcer with active bleeding.

It is clear that a high rebleeding rate occurs in type Ia ulcers (Figure 2) with endoscopic treatment for peptic ulcer bleeding [16]. Failure of endoscopic hemostasis needing further salvage therapy focuses on mortality and successful hemostasis. Thus, mortality rate and rebleeding are important concerns. Most published papers address the technique of TAE and suggest 24-hour availability of an experienced radiologist. Many institutions including our hospital cannot offer this service. Therefore, we perform further analysis if the ulcer type influences the mortality and success rate. Although no significant difference in mortality rate is detected between the sur- 
gery and TAE groups, the rebleeding rate is higher in the TAE group, especially in patients with type Ia PUB. In such patients, the rebleeding rate reached $56.3 \%$ in the TAE group versus only $30.2 \%$ in the surgery group. This difference was not significant, although the TAE group had a higher mortality rate $(p=0.001)$. This contributed to the high total mortality rate (68.6\%) in the patients who suffered from rebleeding after the first salvage therapy despite a second salvage attempt.

Despite the retrospective nature of this study, the results show that the management of PUB refractory to endoscopic treatment is a difficult endeavor with controversial results. The absence of a 24-hour radiology service led by experienced radiologists represents a challenge for many institutions and may have affected the results of the present study. A well-coordinated multidisciplinary team is clearly required for the successful management of this life-threatening condition. Although some studies suggest TAE is a viable alternative to emergency surgery and may even be used as the first-line approach, some factors affecting the success rates require further investigation, such as the lower incidence of rebleeding after surgery in patients with type Ia PUB.

In conclusion, our findings revealed no difference of mortality and rebleeding in patients with type Ia lesions between groups, although rebleeding had a high mortality. We therefore suggest that TAE may be used first in patients with PUB refractory to endoscopic therapy, even in institutions like us with the limitation of no 24-hour availability of an experienced radiologist, whereas emergency surgery may be considered as an alternative treatment in patients with type Ia bleeding in institutions that have no 24-hour radiology service with an experienced radiologist. We look forward more prospective and randomized studies to compare these two methods in such patients.

\section{Acknowledgements}

We are grateful to Nurse Specialist Chang-Chia Chun for data collection and Nurse Specialist Chia-En Hsieh for data management and statistical calculations.

\section{References}

[1] Rockall, T.A., Logan, R.F., Devlin, H.B., et al. (1995) Incidence of and Mortality from Acute Upper Gastrointestinal Haemorrhage in the United Kingdom. Steering Committee and Members of the National Audit of Acute Upper Gastrointestinal Haemorrhage. British Medical Journal, 311, 222-226. http://dx.doi.org/10.1136/bmj.311.6999.222

[2] Lau, J.Y.W., Sung, J.J., Lam, Y.H., et al. (1999) Endoscopic Retreatment Compared with Surgery in Patients with Recurrent Bleeding after Initial Endoscopic Control of Bleeding Ulcers. The New England Journal of Medicine, 340, 751756. http://dx.doi.org/10.1056/NEJM199903113401002

[3] Gisbert, J.P., Calvet, X., Cosme, A., et al. (2012) Long-Term Follow-Up of 1000 Patients Cured of Helicobacter pylori Infection Following an Episode of Peptic Ulcer Bleeding. The American Journal of Gastroenterology, 107, 1197-1204. http://dx.doi.org/10.1038/ajg.2012.132

[4] Lai, K.C., Hui, W.M., Wong, W.M., et al. (2000) Treatment of Helicobacter pylori in Patients with Duodenal Ulcer Hemorrhage-A Long-Term Randomized, Controlled Study. The American Journal of Gastroenterology, 95, 22252232.

[5] Langer, I., Langer, S., Partecke, L.I., et al. (2008) Acute Upper Gastrointestinal Hemorrhage: Is a Radiological Interventional Approach an Alternative to Emergency Surgery? Emergency Radiology, 15, 413-419. http://dx.doi.org/10.1007/s10140-008-0736-Z

[6] Wong, T., Wong, K.T., Chiu, P., et al. (2011) A Comparison of Angiographic Embolization with Surgery after Failed Endoscopic Hemostasis to Bleeding Peptic Ulcer. Gastrointestinal Endoscopy, 73, 900-908. http://dx.doi.org/10.1016/j.gie.2010.11.024

[7] Ang, D., Teo, E.K., Tan, A., et al. (2012) A Comparison of Surgery versus Transcatheter Angiographic Embolization in the Treatment of Nonvariceal Upper Gastrointestinal Bleeding Uncontrolled by Endoscopy. European Journal of Gastroenterology \& Hepatology, 24, 929-938. http://dx.doi.org/10.1097/MEG.0b013e32835463bc

[8] Loffroy, R., Guiu, B., Cercueil, J.P., et al. (2008) Refractory Bleeding from Gastroduodenal Ulcers. Journal of Clinical Gastroenterology, 42, 361-367. http://dx.doi.org/10.1097/MCG.0b013e3180319177

[9] Ripoll, C., Bañares, R., Beceiro, I., et al. (2004) Comparison of Transcatheter Arterial Embolization and Surgery for Treatment of Bleeding Peptic Ulcer after Endoscopic Treatment Failure. Journal of Vascular and Interventional Radiology, 15, 447-450. http://dx.doi.org/10.1097/01.RVI.0000126813.89981.B6

[10] Barkun, A.N., Bardou, M., Kuipers, E.J., et al. (2010) International Consensus Recommendations on the Management of Patients with Nonvariceal Upper Gastrointestinal Bleeding. Annals of Internal Medicine, 152, 101-113. 
http://dx.doi.org/10.7326/0003-4819-152-2-201001190-00009

[11] Holme, J.B., Nielsen, D.T., Funch-Jensen, P., et al. (2006) Transcatheter Arterial Embolization in Patients with Bleeding Duodenal Ulcer: An Alternative to Surgery. Acta Radiologica, 47, 244-247. http://dx.doi.org/10.1080/02841850600550690

[12] Eriksson, L.G., Ljungdahl, M., Sundbom, M., et al. (2008) Transcatheter Arterial Embolization versus Surgery in the Treatment of Upper Gastrointestinal Bleeding after Therapeutic Endoscopy Failure. Journal of Vascular and Interventional Radiology, 19, 1413-1418. http://dx.doi.org/10.1016/j.jvir.2008.06.019

[13] Larssen, L., Moger, T., Bjørnbeth, B.A., et al. (2008) Transcatheter Arterial Embolization in the Management of Bleeding Duodenal Ulcer: A 5.5-Year Retrospective Study of Treatment and Outcome. Scandinavian Journal of Gastroenterology, 43, 217-222. http://dx.doi.org/10.1080/00365520701676443

[14] Loffory, R., Rao, P., Ota, S., et al. (2010) Embolization of Acute Nonvariceal Upper Gastrointestinal Hemorrhage Resistant to Endoscopic Treatment: Results and Predictors of Recurrent Bleeding. CardioVascular and Interventional Radiology, 33, 1088-1100. http://dx.doi.org/10.1007/s00270-010-9829-7

[15] Walsh, R.M., Anain, P., Geisinger, M., et al. (1999) Role of Angiography and Embolization for Massive Gastroduodenal Hemorrhage. Journal of Gastrointestinal Surgery, 3, 61-65. http://dx.doi.org/10.1016/S1091-255X(99)80010-9

[16] Hadžibulić, E. and Govedarica, S. (2007) Significance of Forrest Classification, Rockall's and Blatchford’s Risk Scoring System in Prediction of Rebleeding in Peptic Ulcer Disease. Acta Medica Medianae, 46, 38-43.

[17] Schoenberg, M.H. (2001) Surgical Therapy for Peptic Ulcer and Nonvariceal Bleeding. Langenbeck's Archives of Surgery, 386, 98-103. http://dx.doi.org/10.1007/s004230100210

[18] Loffroy, R., Guiu, B., Mezzetta, L., et al. (2009) Short and Long-Term Results of Transcatheter Embolization for Massive Arterial Hemorrhage from Gastroduodenal Ulcers Not Controlled by Endoscopic Hemostasis. Canadian Journal of Gastroenterology, 23, 115-120. http://dx.doi.org/10.1155/2009/795460

[19] Eriksson, L.G., Sundborm, M., Gustavsson, S., et al. (2006) Endoscopic Marking with a Metallic Clip Facilitates Transcatheter Arterial Embolization in Upper Peptic Ulcer Bleeding. Journal of Vascular and Interventional Radiology, 17, 959-964. http://dx.doi.org/10.1097/01.RVI.0000223719.79371.46 